

\title{
Correlations in p-p Collisions
}

\author{
R. Jefferson Porter* and Thomas A. Trainor (STAR Collaboration) \\ University of Washington \\ E-mail: trainor@hausdorf.npl.washington.edu
}

We present a comprehensive survey of two-particle correlations from p-p collisions at $\sqrt{s}=200$ $\mathrm{GeV}$. Correlations on transverse rapidity $y_{t}$ are separated into a soft component (longitudinal fragmentation) and a hard component (transverse fragmentation). Fragment correlations on transverse rapidity $y_{t}$ are compared to fragmentation functions from $e^{+}-e^{-}$collisions plotted on conventional logarithmic momentum variable $\xi_{p}$ and rapidity $y$. Cuts on transverse rapidity isolate p-p angular correlations on $(\eta, \phi)$ for the two components. Hard-component angular correlations are compared to results from trigger-particle jet analysis. Angular correlations for low- $p_{t}$ fragments, corresponding to low- $Q^{2}$ parton scattering, reveal a large asymmetry about the jet thrust axis.

Correlations and Fluctuations in Relativistic Nuclear Collisions

July 7-9 2006

Florence, Italy

\footnotetext{
${ }^{*}$ Speaker.
} 


\section{Introduction}

QCD theory has predicted abundant production of low- $Q^{2}$ scattered gluons (minijets) in ultrarelativistic nuclear collisions. Such gluons are believed to drive formation of the colored medium in heavy ion collisions at RHIC $[1,2]$. If so, we may discover remnants of low- $Q^{2}(Q \sim 2-10$ $\mathrm{GeV}$ ) partons in the correlation structure of final-state hadrons. Initial studies of correlations in p-p collisions emphasized angle subspace $(\eta, \phi)$ (pseudorapidity and azimuth) [3] and interpreted the results in terms of string fragmentation [4]. Angular correlations from hard-scattered partons (jets) were observed at larger $p_{t}$ and with increasing $\sqrt{s}$ [5]. A two-component model of $\mathrm{p}$-p collisions, with longitudinal and transverse (parton) fragmentation forming distinguishable soft and hard components, describes particle multiplicity distributions at large $\sqrt{s}$ [6].

Two issues arise in a conventional study of jets: the conditional distribution of fragment momenta (fragmentation function) and their angular distribution relative to the parton momentum. The parton momentum can be estimated from hadrons associated with the scattered parton (reconstructed jet), or a high- $p_{t}$ 'leading' or 'trigger' particle. In this analysis we adopt no jet or trigger condition. We measure two-particle correlations on transverse rapidity space $\left(y_{t 1}, y_{t 2}\right)$ to obtain fragment distributions (not fragmentation functions) for a minimum-bias parton sample and on angle space $\left(\eta_{1}, \eta_{2}, \phi_{1}, \phi_{2}\right)$ to obtain corresponding fragment angular correlations. Particle pairs are treated symmetrically, as opposed to asymmetric 'trigger' and 'associated' particle combinations in a conventional high- $p_{t}$ analysis.

Minimum-bias hard-component correlations, in contrast to jet correlations obtained with a trigger particle, represent the majority of parton fragment pairs, those with $p_{t 1} \sim p_{t 2} \sim 1 \mathrm{GeV} / \mathrm{c}$. Symmetric analysis of nonperturbative low- $Q^{2}$ parton fragments requires generalized treatment of fragmentation functions and angular correlations, including use of transverse rapidity $y_{t}$ rather than momentum $p_{t}$, and 2D angular autocorrelations. The more general analysis reveals new information about parton scattering and fragmentation, including angular correlations strongly asymmetric about the jet thrust axis for low- $Q^{2}$ parton collisions. To better understand QCD in A-A collisions we should revisit elementary collisions where novel phenomena are still emerging.

\section{2. $y_{t}$ Spectra}

A recent study of $p_{t}$ spectra from non-single-diffractive (NSD) p-p collisions at $\sqrt{s}=200$ $\mathrm{GeV}$ [7] found that spectra can be separated into a soft component (longitudinal fragmentation) described by a Lévy distribution on transverse mass $m_{t}$ and a hard component (transverse fragmentation) described by a gaussian distribution on transverse rapidity $y_{t}$. The analysis was based on $p_{t}$ spectrum variation with event multiplicity $n_{c h}$. The (semi-)hard component was interpreted as fragments from minimum-bias (mainly low- $Q^{2}$ ) partons.

In Fig. 1 (first panel) we show spectra from ten multiplicity classes $(1, \cdots, 8,9+10,11+12)$ normalized to unit integral when extrapolated to $p_{t}=0$ and plotted on transverse rapidity $y_{t}=$ $\ln \left\{\left(m_{t}+p_{t}\right) / m_{0}\right\}$ with $m_{0} \rightarrow m_{\pi}$ for unidentified hadrons. Transverse rapidity $y_{t}$ provides a common basis, with longitudinal rapidity $y_{z}$, for comparing longitudinal and transverse parton fragmentation. When soft-component model $S_{0}$ (dash-dot curve), defined as the limiting spectrum for $n_{c h} \rightarrow 0$ and modeled by a Lévy distribution on $m_{t}$, is subtracted from the $y_{t}$ spectra we obtain 
distributions in Fig. 1 (second panel) described by hard-component model $H_{0}$ (solid curves), with a gaussian shape on $y_{t}$ essentially independent of $n_{c h}$.
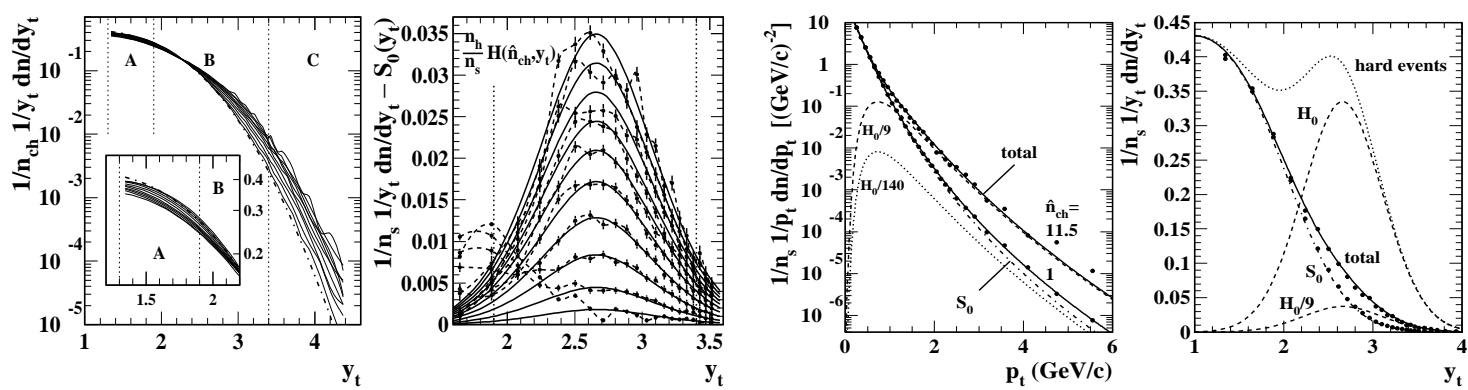

Figure 1: $y_{t}$ spectra for ten multiplicity classes from p-p collsions at $200 \mathrm{GeV}$; corresponding hard components of p-p $y_{t}$ spectra $v s n_{c h}$; $p_{t}$ spectra for $n_{c h}=2$ and $23(|\eta| \leq 0.5)$ with corresponding two-component model functions $S_{0}$ and $H_{0}$ for the two cases; the same data and model functions plotted on $y_{t}$, along with the dotted curve representing minimum-bias p-p collisions with a hard parton scatter.

The conventional power-law model of $p_{t}$ spectra [8] was falsified by these high-statistics p-p data [7]. The two-component spectrum model reveals that $n_{c h}$ selects the frequency of occurrence of minimum-bias parton scattering, dominated by low- $Q^{2}$ scatters. In Fig. 1 (third panel) the relative amplitude of the hard component increases as $n_{c h}$ increases, but the shape does not change. Plotted on $p_{t}$ the spectrum seems to rise in the high- $p_{t}$ region according to the conventional power-law description, but the appearance is deceptive, as shown by the underlying hard-component contribution $H_{0}$ for the two $n_{c h}$ values.

The same data transformed to $y_{t}$ in the fourth panel provide a clearer picture. The soft component is well modeled by an error function on $y_{t}$. The hard component appears as a gaussian, with amplitude depending on the fraction of p-p collisions containing a hard parton collision, in turn determined by the selected $n_{c h}$. The dotted curve estimates the spectrum shape for those NSD collisions with (semi)hard parton scatters (hard p-p collisions). In those collisions, especially in a region of $(\eta, \phi)$ where the parton fragments are localized, the hard component dominates the particle distribution. The single-particle results strongly suggest that two-particle correlations on $\left(y_{t}, y_{t}\right)$ should reveal fragment structure from low- $Q^{2}$ parton collisions inaccessible with conventional jet-finding or trigger-particle methods.

\section{Low- $Q^{2}$ partons in p-p collisions - initial survey of correlations}

The novel result in Fig. 1 (second panel) motivated a follow-up study of two-particle correlations on transverse rapidity in $200 \mathrm{GeV}$ p-p collisions [9, 10]. The minimum-bias distribution on $\left(y_{t}, y_{t}\right)$ in Fig. 2 (first panel) represents all event multiplicities and charge combinations within the STAR $(\eta, \phi)$ detector acceptance. Separate soft and hard components are evident (two peaks), as is the correspondence with the hard-component gaussian $H_{0}$ in the single-particle analysis. The $\left(y_{t}, y_{t}\right)$ space can thus be used as a cut space to study trends of corresponding angular correlations on pseudorapidity $\eta$ and azimuth $\phi$ for soft (longitudinal fragmentation) and hard (transverse or parton fragmentation) components, as shown in the second $\left(p_{t}<0.5 \mathrm{GeV} / \mathrm{c}\right)$ and third $\left(p_{t}>0.5\right.$ $\mathrm{GeV} / \mathrm{c}$ ) panels of Fig. 2. 
A sequence of analysis steps has lead from study of single-particle $p_{t}$ spectra in a twocomponent context to isolation of soft and hard spectrum components to corresponding two-particle correlations on transverse rapidity to jet-like angular correlations obtained without imposition of a jet hypothesis or trigger condition. We thus achieve for the first time a model-independent, minimum-bias analysis of parton scattering and fragmentation. The angular autocorrelations shown in the right panels below provide the first access to jets from low- $Q^{2}$ parton scattering. The lower limit on fragment $p_{t}$ is determined by the fragmentation process itself, not the analysis method. We observe jet correlations in $\mathrm{p}-\mathrm{p}$ collisions down to $p_{t}=0.35 \mathrm{GeV} / \mathrm{c}$ for both hadrons. The main subject of this paper is the properties of fragment distributions on rapidity and angle from low- $Q^{2}$ partons in $\mathrm{p}-\mathrm{p}$ collisions.
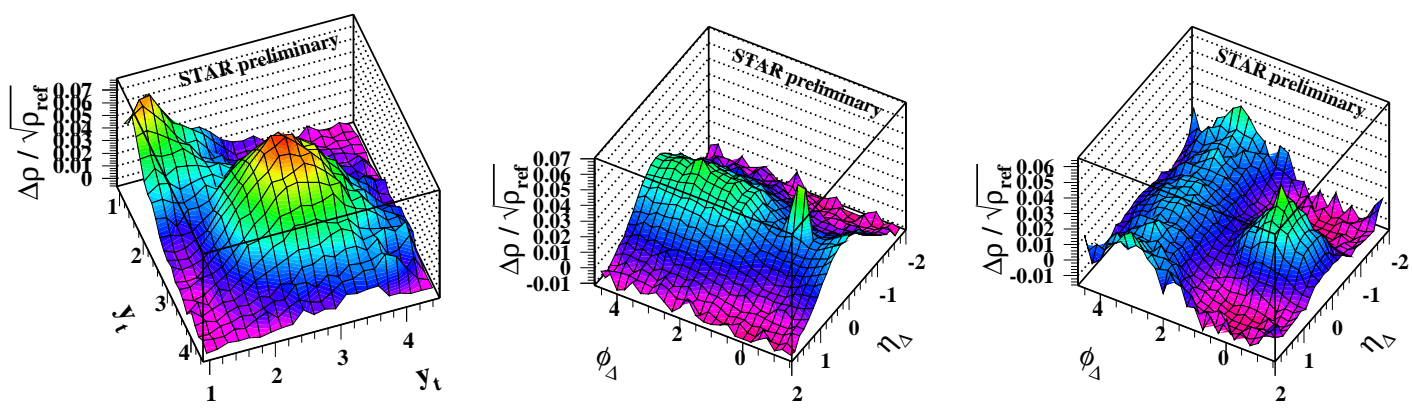

Figure 2: Longitudinal (soft) and transverse (hard) fragment correlations on $\left(y_{t 1}, y_{t 2}\right)$; soft fragment angular correlations on difference axes $\left(\eta_{\Delta}, \phi_{\Delta}\right)$; hard fragment angular correlations.

\section{Correlation analysis method}

The underlying principle of the analysis method is simple: from 6D two-particle momentum space $\left(y_{t 1}, \eta_{1}, \phi_{1} ; y_{t 2}, \eta_{2}, \phi_{2}\right)$ we project to $2 \mathrm{D}$ subspaces with as little distortion and loss of information as possible. The first step is a partition to transverse-rapidity and angular subspaces $\left(y_{t 1}, y_{t 2}\right)$ and $\left(\eta_{1}, \phi_{1}, \eta_{2}, \phi_{2}\right)$. The minimum-bias distribution on $\left(y_{t 1}, y_{t 2}\right)$ (integral over angular acceptance) is shown in Fig. 2 (first panel). The quantity on the vertical axis is discussed below. The two peaks are labeled soft (smaller $y_{t}$ ) and hard (larger $y_{t}$ ) components.

Reduction of $\left(\eta_{1}, \phi_{1}, \eta_{2}, \phi_{2}\right)$ to a $2 \mathrm{D}$ space with minimal distortion relies on the autocorrelation concept and stationarity $[11,12]$. Space $\left(\eta_{1}, \eta_{2}\right)$ for example can be rotated to sum and difference axes $\left(\eta_{\Sigma}, \eta_{\Delta}\right)$. Near mid-rapidity $(\eta=0)$ correlations are typically slowly varying or constant on sum axis $\eta_{\Sigma} \equiv \eta_{1}+\eta_{2}$ [12]. Thus, all significant structure lies on difference axis $\eta_{\Delta} \equiv \eta_{1}-\eta_{2}$. Averaging the two-particle density over $\eta_{\Sigma}$ results in an autocorrelation on $\eta_{\Delta}$ with no information loss. A similar simultaneous average on $\phi_{\Sigma}$ results in a $2 \mathrm{D}$ angular autocorrelation on $\left(\eta_{\Delta}, \phi_{\Delta}\right)$, as in Fig. 2 (right two panels). Those distributions can be separated into same-side (SS, $\phi_{\Delta}<\pi / 2$ ) and away-side (AS, $\phi_{\Delta}>\pi / 2$ ) components.

Correlation measure $\Delta \rho / \sqrt{\rho_{\text {ref }}}$ is closely related to Pearson's correlation coefficient or normalized covariance [13]. For event-wise particle sums $n_{a}$ and $n_{b}$ in histogram bins $a$ and $b$ on single-particle space $x$ Pearson's normalized covariance is $r_{a b} \equiv \overline{(n-\bar{n})_{a}(n-\bar{n})_{b}} / \sqrt{\overline{(n-\bar{n})_{a}^{2}} \overline{(n-\bar{n})_{b}^{2}}}$ averaged over the event ensemble. The closely-related covariance density ratio is $\Delta \rho / \sqrt{\rho_{\text {ref }}} \equiv$ 
$1 / \varepsilon_{x} \cdot \overline{(n-\bar{n})_{a}(n-\bar{n})_{b}} / \sqrt{\bar{n}_{a} \bar{n}_{b}}$, where $\varepsilon_{x}$ is the histogram bin size on $x$ and Poisson values of the number variances in the denominator are substituted. $\Delta \rho / \sqrt{\rho_{\text {ref }}}$, estimating the density of correlated pairs per particle, is our correlation measure for two-particle distributions on $\left(y_{t 1}, y_{t 2}\right)$, $\left(\eta_{1}, \eta_{2}\right),\left(\phi_{1}, \phi_{2}\right)$ and $\left(\eta_{\Delta}, \phi_{\Delta}\right)$.

We obtain angular autocorrelations on $\left(\eta_{\Delta}, \phi_{\Delta}\right)$ ( $c f$. Fig. 2 , right panels) by averaging $\Delta \rho / \sqrt{\rho_{\text {ref }}}$ over diagonal strips on $\left(\eta_{1}, \eta_{2}\right)$ and $\left(\phi_{1}, \phi_{2}\right)$ defined by bins on $\left(\eta_{\Delta}, \phi_{\Delta}\right)$ [equivalently, averaging over bin indices $(a, b)$ with $a-b=k][11] . \Delta \rho / \sqrt{\rho_{\text {ref }}}$ is ideally suited for tests of linear superposition, e.g., comparing A-A collisions to linear superpositions of $\mathrm{p}-\mathrm{p}$ collisions. Conventional "correlation function" $\Delta \rho / \rho_{\text {ref }}$ (e.g., HBT analysis) is a per-pair measure containing a trivial $1 / n$ factor which distracts from substantive physics issues.

\section{Fragmentation functions in $e^{+}-e^{-}$collisions}

Our primary focus is parton fragmentation in p-p collisions. However, to better interpret the p-p results we consider fragmentation in $e^{+}-e^{-}$collisions where the connection to QCD theory is well established [14]. A fragmentation function (FF) describes the conditional distribution of fragment momenta given the parton momentum (estimated from collision kinematics or the observed fragment distribution or jet). Fragment momenta can be normalized by the parton momentum (e.g., $x_{p}=p_{\text {fragment }} / p_{\text {parton }}$, with $p_{\text {parton }}$ determined by jet reconstruction, by $\sqrt{s} / 2\left(\mathrm{e}^{+}-\mathrm{e}^{-}\right)$or by $Q / 2$ (e-p deep-inelastic scattering or DIS). Alternatively, relative momentum can be measured logarithmically by $\xi_{p} \equiv \ln \left(1 / x_{p}\right)$. In [14] fragment and parton momenta are represented by rapidity $y=\ln \left\{(E+p) / m_{0}\right\}$, where $m_{0}$ is the fragment mass (pion mass for unidentified hadrons). Then $\xi_{p} \sim y_{\text {parton }}-y_{\text {fragment }}$.
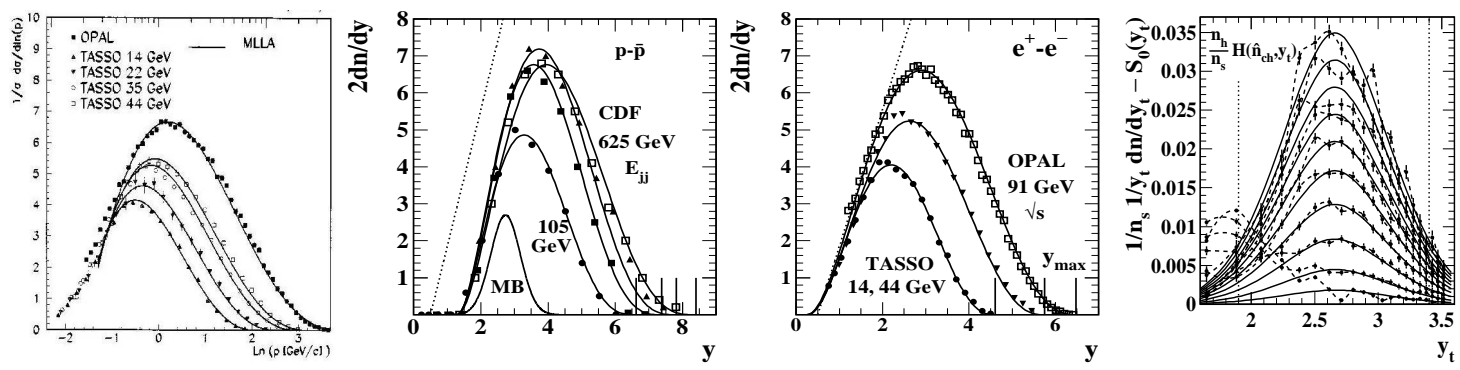

Figure 3: $e^{+}-e^{-}$fragmentation functions from TASSO and OPAL plotted on $\ln (p)$; CDF fragmentation functions plotted on rapidity $y$; TASSO and OPAL fragmentation functions plotted on rapidity $y$ with beta distributions (solid curves); hard components from STAR p-p $p_{t}$ spectra plotted on $y_{t} v s n_{c h}$.

In Fig. 3 we plot fragmentation functions from several collisions systems $[15,16,17]$. In the first panel we show FFs on $\log (p)$ from $e^{+}-e^{-}$collisions at $\sqrt{s}=14,22,35$ and $44 \mathrm{GeV}[16]$ and 91 $\mathrm{GeV}$ [17]. The solid curves are QCD theory (modified leading-log approximation or MLLA [18]) and reflect momentum conservation and QCD branching above the peaks. In the second panel

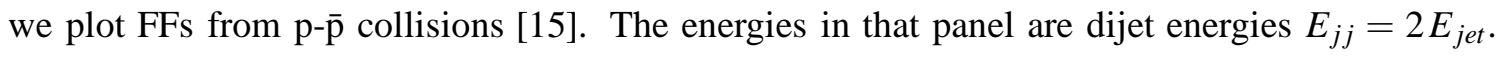
The hard component from the STAR two-component analysis of p-p collisions (fourth panel) [7] is included schematically as the small gaussian curve (MB: minimum-bias parton fragments with no $y_{\max }$ condition). In the third panel we plot select data from the first panel on rapidity $y$. 
FFs plotted on rapidity are well described by the beta distribution $\beta(x ; p, q)=x^{p-1}(1-$ $x)^{q-1} / B(p, q)$, with beta function $B(p, q) \equiv \Gamma(p+q) / \Gamma(p) \Gamma(q)$. In contrast to the MLLA curves in the first panel the beta distributions in the third panel provide a precise description of the entire fragment distribution. The beta distribution defined on $x \in[0,1]$ is in each case rescaled to $\beta(y ; p, q)$ on $\left[y_{\min }, y_{\max }\right] . y_{\min }$ is $0.35(p \sim 0.08 \mathrm{GeV} / \mathrm{c})$ for e-e collisions and $1.5(p \sim 0.35 \mathrm{GeV} / \mathrm{c})$ for $\mathrm{p}$-p collisions. The parton momentum is represented by $y_{\max }\left(X ; m_{0}\right) \equiv \ln \left\{\left(\sqrt{X^{2}+m_{0}^{2}}+X\right) / m_{0}\right\}$, with $X=E_{\text {jet }}, \sqrt{s} / 2$ or $Q / 2$ depending on the collision system. $y_{\max }$ values for each fragmentation function in Fig. 3 (center panels) are marked by vertical lines. The energy systematics of beta distribution parameters for $e^{+}-e^{-}$collisions is presented in [14]. In the fourth panel we repeat the hard-component data from $200 \mathrm{GeV}$ p-p collisions, corresponding to parton collisions at energy scales of a few $\mathrm{GeV}$ which are similar in form to FFs for $Q \sim 100 \mathrm{GeV}$.

\section{6. p-p correlations on $\left(y_{t}, y_{t}\right)$}

Given the phenomenology of fragmentation functions from jets at large energy scales we now return to correlations in p-p collisions on $\left(y_{t}, y_{t}\right)$. Particle pairs from nuclear collisions can be separated on azimuth into same-side (SS) and away-side (AS) pairs. Fig. 4 shows SS (left panels) and AS (right panels) correlations of the form $\Delta \rho / \sqrt{\rho_{\text {ref }}}$ on $\left(y_{t 1}, y_{t 2}\right)$. Each pair of panels represents like-sign (LS) and unlike-sign (US) charge combinations (left and right respectively). Structure can be separated into a soft component $\left(y_{t}<2\right.$ or $p_{t}<0.5 \mathrm{GeV} / \mathrm{c}$ for each particle) and a hard component $\left(y_{t}>2\right.$ for each particle). Both components are strongly dependent on charge-sign combination (LS and US) and $\phi_{\Delta}$ (SS and AS). Description of $\left(y_{t}, y_{t}\right)$ structure is simplest in terms of sum and difference axes $y_{t \Sigma} \equiv y_{t 1}+y_{t 2}$ and $y_{t \Delta} \equiv y_{t 1}-y_{t 2}$.
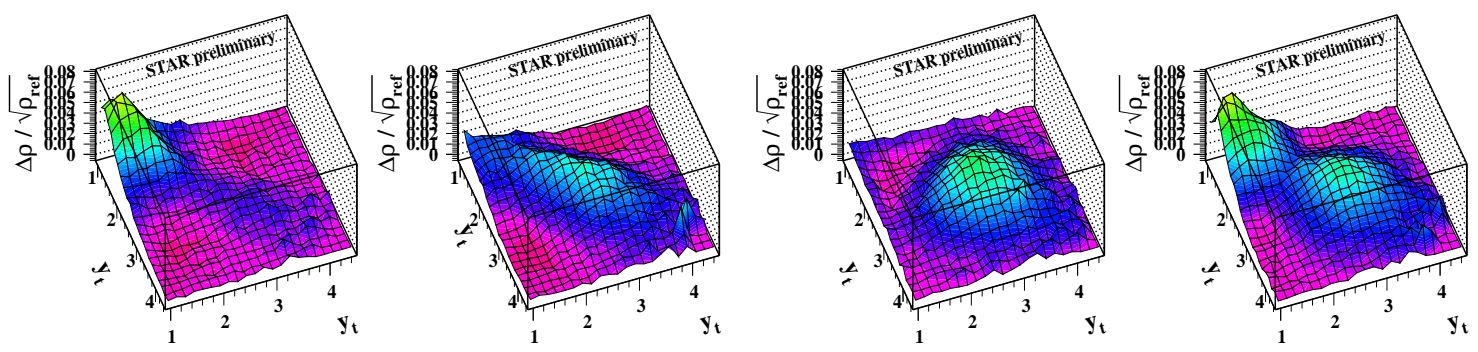

Figure 4: Correlations on transverse rapidity $\left(y_{t}, y_{t}\right)$ from longitudinal and transverse fragmentation and from quantum correlations (HBT, SS-LS) for same-side $\phi_{\Delta}<\pi / 2$ (left) and away-side $\phi_{\Delta}>\pi / 2$ (right) pairs, and for like-sign and unlike-sign charge combinations respectively in each pair of panels.

In the left (SS) panels, the LS soft component (first panel) is interpreted as quantum correlations (HBT). The LS hard component along the diagonal is small and may itself be dominated by quantum correlations (from parton fragmentation). The US hard component (second panel) is a peak at $y_{t} \sim 2.8\left(p_{t} \sim 1 \mathrm{GeV} / \mathrm{c}\right)$ elongated along $y_{t \Sigma}$. The hard component runs continuously into the US soft component at lower $y_{t}$, which is suppressed (relative to AS-US pairs) due to transverse-momentum conservation. The SS-US hard component represents the first measurement of minimum-bias intra-jet rapidity correlations in nuclear collisions. In the right (AS) panels, the hard-component peaks for LS and US pairs have similar shapes and amplitudes, are nearly sym- 
metric about their centers and much broader on $y_{t \Delta}$ than their SS-US counterpart, with rapid falloff below $y_{t \Sigma} \sim 4$ (hadron $p_{t} \sim 0.5 \mathrm{GeV} / \mathrm{c}$ ).

The large US soft component (fourth panel) represents longitudinal fragmentation, which is dominated by local momentum and charge conservation and therefore contributes negligibly to ASLS correlations (third panel). The conventional description of longitudinal fragmentation is 'string' fragmentation [4]. We don't observe the next-to-nearest-neighbor soft LS correlations displaced from the $\eta_{\Delta}$ origin that charge-ordered string fragmentation would imply. Measured correlations are more compatible with a picture of small-angle (longitudinal) gluon scattering and fragmentation to one or two pions from those gluons which appear in the detector rapidity acceptance. Away-side transverse parton fragmentation is independent of charge combination.

\section{7. $\left(y_{t}, y_{t}\right)$ intra-jet correlations and QCD}

To study two-particle fragment correlations from low- $Q^{2}$ partons we must symmetrize the analyses on $y_{t}$ and $(\eta, \phi)$. Symmetrized same-side (SS) unlike-sign (US) correlations on $\left(y_{t}, y_{t}\right)$ in Fig. 4 (second panel) comprise a two-particle intra-jet fragment distribution. We now model that distribution based on our phenomenological description of parton fragmentation in $e^{+}-e^{-}$collisions [14]. We combine information from single-particle FFs with expectations for two-particle correlations to sketch a two-particle fragment distribution and consider how it relates to QCD.
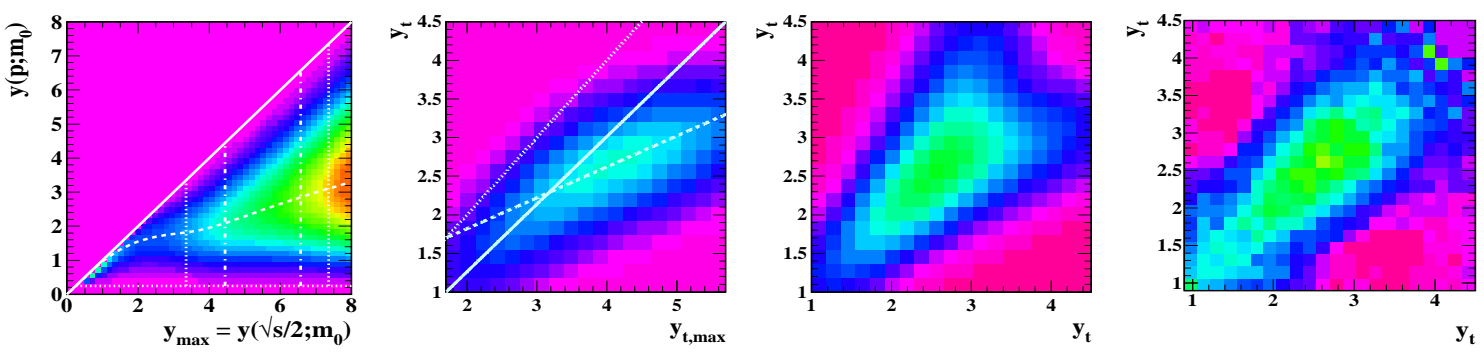

Figure 5: Joint fragment distribution on $\left(y, y_{\max }\right)$ (fragment rapidity vs parton rapidity) representing all data from $e^{+}-e^{-}$collisions at PETRA and LEP; sketch of corresponding distribution for low- $Q^{2}$ partons in $\mathrm{p}-\mathrm{p}$ collisions; symmetrized distribution for fragment-fragment correlations; measured intra-jet correlations.

In Fig. 5 (first panel) we show the joint distribution on fragment and parton rapidity $\left(y, y_{\max }\right)$ which represents all $e^{+}-e^{-}$fragmentation functions from $10-200 \mathrm{GeV}$ to the statistical accuracy of the data [14]. The extrapolated trends near the origin are most relevant to the problem of low- $Q^{2}$ parton fragmentation in nuclear collisions. In the second panel we show a sketch based on the first panel but more appropriate to two-particle correlations. The sketch incorporates an underlying parton energy distribution which falls off rapidly with increasing $y_{\max }$. The dotted line is the kinematic trend expected at low $Q^{2}$ for gluon $\rightarrow$ one hadron. The solid line is the kinematic trend for fragmentation to two hadrons, the limiting case for two-particle correlations. The dashed line is the 'locus of modes' (most probable points) consistent with FFs from high-energy $e^{+}-e^{-}$collisions.(dashed curve in first panel) Our model function (2D histogram) follows the locus of modes at larger $y_{t, \max }$ but transitions to the solid line consistent with the limiting constraint of two fragments for smaller parton momenta. The distribution width is consistent with FFs on $y$ [14] and the p-p $p_{t}$ spectrum 
hard component $H_{0}$ [7]. A vertical slice from that distribution for a given $y_{t, \max }$ is a fragmentation function representing at least two fragments.

In the third panel we symmetrize the distribution from the second panel about the diagonal, because in our $\left(y_{t}, y_{t}\right)$ analysis we correlate fragments with fragments symmetrically rather than fragments with partons or with special trigger particles. The sketch is compared with SS-US data in the fourth panel - the minimum-bias two-particle intra-jet fragment distribution. For low- $Q^{2}$ partons, which dominate the minimum-bias parton distribution, the two-particle fragment distribution becomes symmetric about the sum diagonal (the most-probable fragment number $\rightarrow 2$ ). The comparison between model function and data is both impressive and informative. The slight excess yield at small $y_{t}$ in the fourth panel is 'longitudinal' fragmentation: emission of low- $p_{t}$ same-side US pairs from longitudinal fragmentation is suppressed in accord with transverse-momentum conservation.

We can compare the soft-component (mainly AS) US correlations on $\left(\eta_{\Delta}, \phi_{\Delta}\right)$ in Fig. 2 (second panel) to the hard-component SS-US correlations on $\left(y_{t}, y_{t}\right)$ in Fig. 4 (second panel). In both cases the distribution is a gaussian on rapidity difference: $y_{t \Delta} v s y_{z \Delta} \rightarrow \eta_{\Delta}$. Given the interpretation of the former in terms of parton fragmentation we conclude that the 'soft' component of the $\left(\eta_{\Delta}, \phi_{\Delta}\right)$ correlations is also fragmentation-low- $Q^{2}$ partons (mainly gluons) fragment to two hadrons after scattering to small angles from participant nucleons. This is the sort of comparison made possible by using transverse rapidity rather than transverse momentum to study fragmentation, even for $p_{t} \sim 1 \mathrm{GeV} / \mathrm{c}$

\section{8. $\left(y_{t}, y_{t}\right)$ intra-jet correlations and trigger-particle analysis}

In this section we emulate the conventional trigger-particle approach to fragmentation functions. In Fig. 6 (first panel) we repeat the minimum-bias $\left(y_{t}, y_{t}\right)$ SS-US pair distribution. The lines and boxes define 'trigger' and 'associated' cut regions used in high- $p_{t}$ trigger-particle analyses. For example, the dashed boxes along the upper-right edges correspond to a trigger particle with $p_{t} \in[4,6] \mathrm{GeV} / \mathrm{c}\left(y_{t} \in[4,4.5]\right)$ and an associated particle with $p_{t} \in[0.15,4] \mathrm{GeV} / \mathrm{c}\left(y_{t} \in[1.2,4]\right)$, as defined in [19].
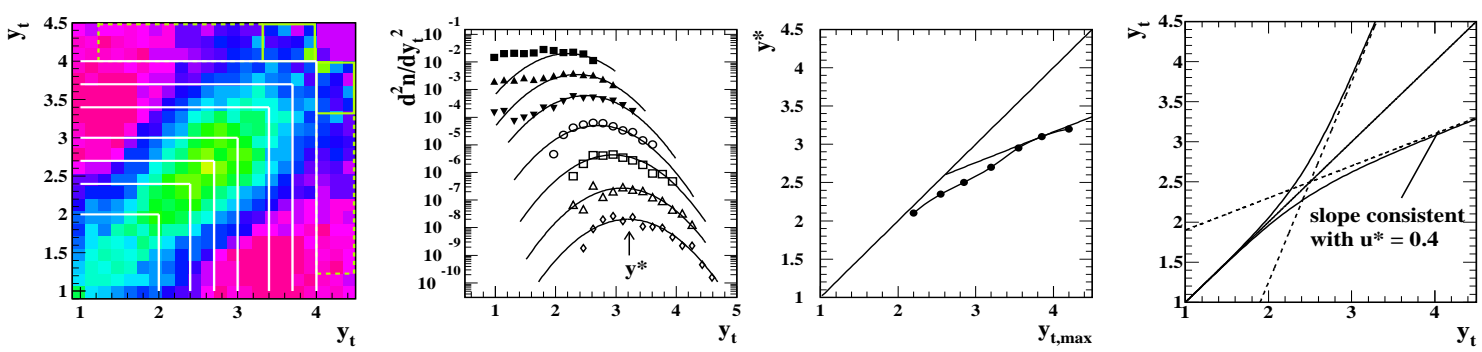

Figure 6: Intra-jet correlations on $\left(y_{t}, y_{t}\right)$ used as a cut space to emulate trigger-particle analysis; conditional fragment distributions inferred from the seven cut regions in the first panel; modes $y^{*}$ of the seven fragment distributions; locus-of-modes parameterization (solid curves) used to produce the middle panels of Fig. 6.

In the second panel we plot the conditional distributions on fragment rapidity $y_{t}$ corresponding to the seven L-shaped regions in the first panel. The solid squares correspond to the first trigger interval $y_{t} \in[2,2.4]$, and the open diamonds correspond to the last trigger interval $y_{t} \in[4,4.5]$ 
(heavy ion collisions). The modes $y^{*}$ of the conditional distributions increase monotonically. The solid curves are gaussians with the same width (0.46) as the hard component $H_{0}$ isolated in $1 \mathrm{D}$ $y_{t}$ spectra shown in Fig. 1 (second panel). By imposing those cuts on $\left(y_{t}, y_{t}\right)$ we bias the parton distribution to larger $y_{\max }$, and the FF mode changes accordingly. The lowest set of data points (open diamonds) is the p-p equivalent of FFs inferred in heavy ion collisions by a conventional trigger-particle analysis [19].

In the third panel we plot peak modes $v s$ trigger rapidity $y_{t, \max }$ (centers of trigger strips in the first panel). The asymptotic slope of the data for larger $y_{t, \max }$ is consistent with $u^{*}=0.4$ derived from $e^{+}-e^{-}$FFs [14]. In the fourth panel we plot the trends which generated the sketch in Fig. 5 (second panel). The FF trends from $e^{+}-e^{-}$collisions and from p-p correlations are compatible.

\section{Angular correlations on $\left(\eta_{\Delta}, \phi_{\Delta}\right)$}

The $\left(y_{t}, y_{t}\right)$ correlations in the previous section are directly related to angular correlations on $(\eta, \phi)$. To isolate soft and hard components of p-p angular correlations we define soft pairs by $y_{t}<2\left(p_{t}<0.5 \mathrm{GeV} / \mathrm{c}\right)$ and hard pairs by $y_{t}>2$ for each particle of a pair. Fig. 7 shows minimumbias correlations (all pairs for all event multiplicity classes) on $\left(\eta_{\Delta}, \phi_{\Delta}\right)$ for the soft component (left panels) and hard component (right panels), with LS and US charge combinations (left and right respectively). The first panel is dominated by a $2 \mathrm{D}$ gaussian peak at the origin representing quantum correlations (HBT). The US combination in the next panel is dominated by a 1D gaussian peak on $\eta_{\Delta}$ arising from local charge conservation during longitudinal fragmentation. That trend is suppressed near the origin (the depression on $\phi_{\Delta}$ of the gaussian on $\eta_{\Delta}$ ) due to local transversemomentum conservation. The narrow peak at the origin is electron-positron pairs from photon conversions. Except for the HBT contribution the structure of the soft component is apparently a consequence of local measure conservation (momentum and charge) during longitudinal fragmentation.
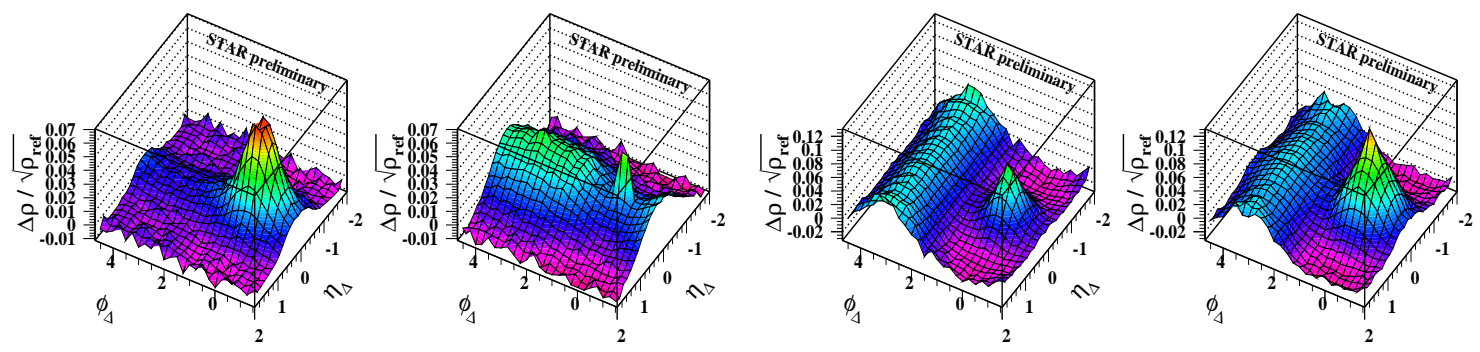

Figure 7: Number correlations on $\left(\eta_{\Delta}, \phi_{\Delta}\right)$ for soft-component (left panels) and hard-component (right panels) pairs, and for like-sign LS and unlike-sign US charge combinations respectively in each panel pair.

The hard-component correlations in the right panels consist of a same-side peak at the origin and an away-side ridge. The SS-LS peak (third panel) may be dominated by quantum correlations rather than jet fragmentation per se. The SS-US peak (fourth panel) represents angular correlations of parton fragments (jet cone). The AS hard-component correlations for LS and US pairs are essentially identical in shape and amplitude and reflect momentum conservation between scattered partons (dijets), including uniformity on $\eta_{\Delta}$ due to the broad distribution of parton-collision centers 
of momentum (broad parton $x$ distribution). Those hard-component angular correlations, qualitatively consistent with conventional expectations for high- $p_{t}$ jet angular correlations, are observed in this study for pairs of particles with both $p_{t}$ s as low as $0.35 \mathrm{GeV} / \mathrm{c}\left(y_{t} \sim 1.6\right)$, much lower than previously observed with trigger-particle methods.

\section{0. $Q^{2}$ dependence of fragmentation and water drop collisions}

Jet structure is characterized by two-particle angular correlations of hadron fragments, both intra-jet (within one jet) and inter-jet (between opposing dijets). In a conventional high- $p_{t}$ triggerparticle analysis angular correlations relative to the trigger reveal 2D same-side peaks with (possibly different) widths on $\eta$ and $\phi$. In our minimum-bias study we encounter fragment distributions with a most-probable $p_{t}$ of $1 \mathrm{GeV} / \mathrm{c}$. The most-probable fragment multiplicity is 2 , with approximately equal fragment momenta, and the most-probable parton energy $Q / 2$ is thus somewhat more than $2 \mathrm{GeV}$. For jets from low- $Q^{2}$ partons we cannot differentiate trigger and associated particles. We focus on the $Q$ dependence of intra-jet angular correlations, with $Q$ determined by the mean of the nearly-symmetric fragment momenta.
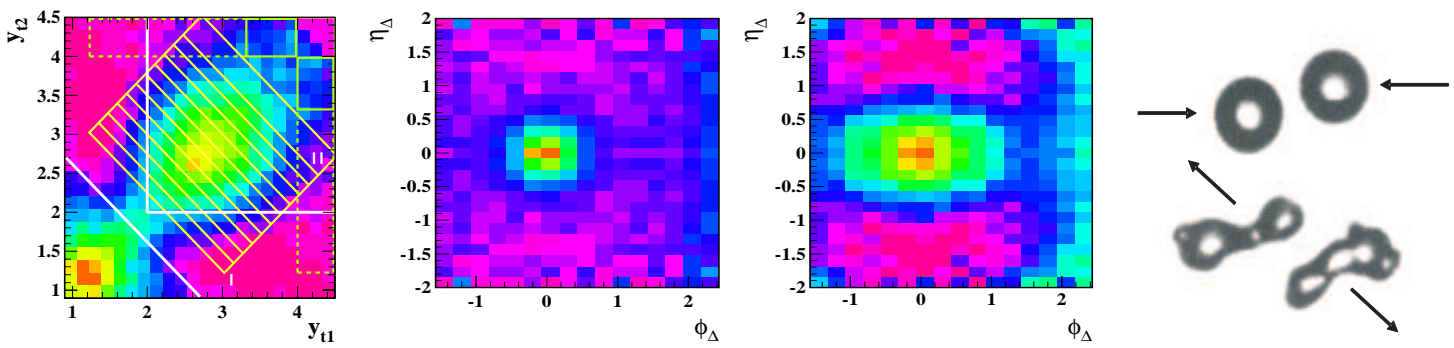

Figure 8: Fragment correlations on $\left(y_{t}, y_{t}\right)$ used as a cut space to study the $Q^{2}$ dependence of fragment angular correlations; unlike-sign angular correlations for bin 11 in the first panel; US angular correlations for bin 1; two frames from a high-speed movie of colliding water drops showing the fragmentation process.

Fig. 8 (first panel) shows minimum-bias (all pairs) two-particle correlations on transverserapidity $\left(y_{t}, y_{t}\right)$. Because of the symmetry the space is best described on sum and difference axes $y_{t \Sigma} \equiv y_{t 1}+y_{t 2}$ and $y_{t \Delta} \equiv y_{t 1}-y_{t 2}$. The parton $Q$ for angular correlations is defined by the grid of rectangles or bins along $y_{t \Sigma}$ numbered $1, \cdots, 11$. The bin labeled 1 corresponds to fragment $p_{t} \sim 0.6$ $\mathrm{GeV} / \mathrm{c}$; the bin labeled 11 corresponds to fragment $p_{t} \sim 2.5 \mathrm{GeV} / \mathrm{c}$. The solid boxes in the upperright corner represent regions defined in an initial high- $p_{t}$ trigger-particle analysis at RHIC [20]. The dashed extensions represent associated-particle cuts later applied to heavy ion collisions [19]. In contrast to the trigger-particle cuts the cuts on $y_{t \Sigma}$ for this study, with large acceptance on $y_{t \Delta}$, are required to avoid biasing low- $Q^{2}$ angular correlations.

The second and third panels of Fig. 8 show angular autocorrelations for the eleventh and first bins on $y_{t \Sigma}$ in the first panel plotted on $\left(\eta_{\Delta}, \phi_{\Delta}\right)$ with a 1:1 aspect ratio. The second panel shows angular correlations for bin 11. The SS peak (jet cone) is narrow, the base radius $\sim 0.7$ being typical for a high- $p_{t}$ leading-particle analysis. The AS ridge on $\phi_{\Delta}$ (not visible, particle pairs from dijets) is uniform on $\eta_{\Delta}$ as expected, due to the broad parton $x$ distribution. The third panel shows angular correlations from $y_{t \Sigma}$ bin 1 , where the most probable combination is two particles each with $p_{t} \sim 0.6 \mathrm{GeV} / \mathrm{c}$. The SS peak is much broader (as is the AS ridge onn $\phi_{\Delta}$ ) and has a large 
eccentricity, but is still well defined. That jet correlation is a remarkable result for particles with such low $p_{t}$ and illustrates the power of the angular autocorrelation technique.

Fragment angular correlations change dramatically with parton energy at low $Q^{2}$. The most remarkable feature of the third panel is the large asymmetry between azimuth and pseudorapidity, a feature not predicted by pQCD. A possible explanation for the asymmetry is pictured in the fourth panel: two frames from a movie of water drops colliding with an intermediate impact parameter [21]. At that impact parameter and speed $(6 \mathrm{~m} / \mathrm{s})$ the colliding drops (upper frame) undergo $2 \rightarrow 4$ fragmentation. They form an expanding disk along the contact plane which fragments in two stages: 1) the disk (evolving to a thickened rim) breaks in half, the halves flying apart in the reaction plane defined by impact parameter and collision axis; 2) the two rim segments elongate normal to the reaction plane and fragment to drop pairs aligned in the azimuth direction (lower frame), as we observe in the third panel. If that model is applicable to low- $Q^{2}$ parton collisions it implies that the projectile partons interact as extended objects, and the fragmentation remembers the impact parameter orientation. In effect, partons at that energy scale are nearly hadrons. That picture is consistent with local parton-hadron duality (LPHD) [22]. Partons at small energy scales (low $Q^{2}$ ) are nearly hadrons but still interact as partially-colored objects. Ironically, low- $x$ and low- $Q^{2}$ parton collisions at RHIC may have hydrodynamic aspects similar to heavy ion collisions at the Bevalac.

\section{Comparison with minijet deformation in heavy ion collisions}

What happens to the fragment angular asymmetry in A-A collisions? A strong asymmetry has also been observed in Au-Au collisions, but the sense of the asymmetry is opposite to (elongation rotated $90^{\circ}$ relative to) that in Fig.8. In Fig. 9 (first panel) we repeat the low- $Q^{2}$ p-p result. In the next panel we show a similar analysis of mid-central Au-Au collisions at $130 \mathrm{GeV}$ [23]. A cos $\left(2 \phi_{\Delta}\right)$ contribution from elliptic flow has been subtracted leaving the SS minijet structure. Both panels are plotted with 1:1 aspect ratios. In the third panel the same distribution is shown in perspective. Note that no away-side jet ridge survives in the more central $\mathrm{Au}-\mathrm{Au}$ collisions, consistent with trigger-particle studies [20].
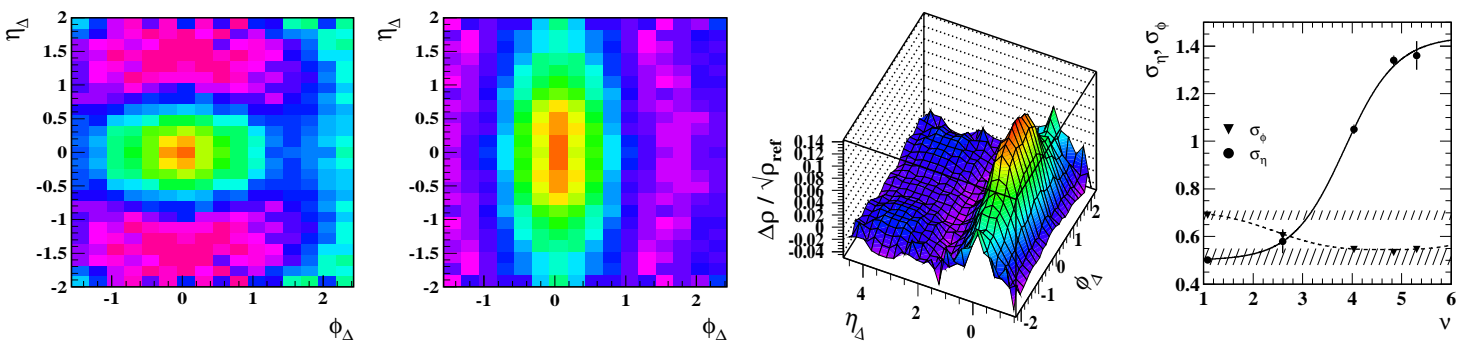

Figure 9: Angular correlations from low- $Q^{2}$ parton fragmentation in p-p collisions ( $c f$. previous figure); minijet angular correlations from $130 \mathrm{GeV}$ mid-central Au-Au collisions (1:1 aspect ratio); same in perspective view; same-side peak width systematics $v s$ collision centrality for $130 \mathrm{GeV} \mathrm{Au}$-Au collisions.

In Fig. 9 (fourth panel) the pseudorapidity (solid) and azimuth (dashed) widths of the sameside peak are plotted $v s$ A-A centrality measured by $v$, which estimates the number of binary $\mathrm{N}-\mathrm{N}$ collisions per participant nucleon pair. We observe that the azimuth width decreases from 
the minimum-bias p-p value with increasing centrality (a novel result), whereas the pseudorapidity width greatly increases, the elongation being obvious in the autocorrelation data (middle panels) [23]. In an A-A collision the parton collisions are 'minimum bias,'- there can be no selection of parton energy scale. Thus, the p-p azimuth width in the fourth panel (solid triangle on the dashed curve at $v=1$ ) is from minimum-bias p-p data, as opposed to the first panel of Fig. 9 where low- $Q^{2}$ parton collisions are selected. What causes the pseudorapidity $\eta$ elongation in A-A collisions? One can speculate that the fragmentation process in heavy ion collisions is coupled to a longitudinally-expanding colored medium, as stated in [23].

\section{Discussion}

We find that all features of p-p correlations at $200 \mathrm{GeV}$ can be explained by a simple model of low- $x$ parton (mainly gluon) scattering at low $Q^{2}$, each gluon fragmenting to one or two hadrons (mainly pions). The observable soft component of the correlations is consistent with small-angle (longitudinal) gluon scattering, leaving one gluon and its fragment(s) in the detector acceptance near mid-rapidity. The minimum-bias hard component is consistent with large-angle (transverse) scattering of two gluons to a total of two, three or four hadrons. Local momentum and charge conservation during scattering and fragmentation dominate the charge and angle dependence. Those basic elements account for all systematic features of the minimum-bias p-p correlation data.

\section{Summary}

We have presented a broad survey of two-particle correlations from $200 \mathrm{GeV}$ p-p collisions at RHIC. Correlations from longitudinal and transverse (parton) fragmentation are clearly distinguished. Parton fragmentation in p-p collisions is precisely accessible down to $p_{t} \sim 0.35 \mathrm{GeV} / \mathrm{c}$ for both hadrons of a correlated pair with new analysis methods based on transverse rapidity and angular autocorrelations. Low- $Q^{2}$ fragmentation systematics are dominated by local momentum and charge conservation. The hard component in this study provides new access to minimum-bias parton scattering and fragmentation.

Jet morphology for low- $Q^{2}$ partons requires a more general treatment of fragment $p_{t}$ distributions and angular correlations. Fragment distributions on transverse rapidity $y_{t}$ are well-behaved at low $p_{t}$ and exhibit interesting systematic behaviors which can be compared with LPHD. Jet angular correlations at low- $Q^{2}$ show a large asymmetry about the thrust axis (up to 2:1 eccentricity favoring the azimuth direction), possibly related to nonperturbative details of low- $Q^{2}$ parton collisions. These measurements of $\mathrm{p}$-p correlations provide an essential reference for the study of in-medium modification of parton scattering and fragmentation in heavy ion collisions.

This work was supported in part by the Office of Science of the U.S. DoE under grant DEFG03-97ER41020.

\section{References}

[1] K. Kajantie, P. V. Landshoff and J. Lindfors, Phys. Rev. Lett. 59, 2527 (1987).

[2] A. H. Mueller, Nucl. Phys. B 572, 227 (2000). 
[3] J. Whitmore, Phys. Rept. 27, 187 (1976);

T. Kafka et al,, Phys. Rev. D 16 1261, (1977).

[4] B. Andersson, G. Gustafson, G. Ingelman and T. Sjöstrand, Phys. Rep. 97, 31 (1983).

[5] A. L. S. Angelis et al. (CCOR), Phys. Lett. B 97, 163 (1980);

C. Albajar et al. (UA1), Nucl. Phys. B 309, 405 (1988).

[6] A. Giovannini and R. Ugoccioni, Phys. Rev. D 59, 094020 (1999).

J. Dias de Deus and R. Ugoccioni, Phys. Lett. B 494, 53 (2000).

[7] J. Adams et al. (STAR Collaboration), Phys. Rev. D 74, 032006 (2006).

[8] C. Albajar et al. (UA1 Collaboration), Nucl. Phys. B 335, 261 (1990).

[9] R. J. Porter and T. A. Trainor (STAR Collaboration) Acta Phys. Polon. B 36, 353 (2005).

[10] T. A. Trainor (STAR Collaboration), Proceedings of the International Symposium on Multiparticle Dynamics (ISMD 2005), Kromêr̂́î, CR, August 9-15, 2005, Published in AIP Conf. Proc. 828, 238 (2006).

[11] T. A. Trainor, R. J. Porter and D. J. Prindle, J. Phys. G 31, 809 (2005); D. J. Prindle and T. A. Trainor, J. Phys. Conf. Ser. 27, 118 (2005).

[12] J. Adams et al. (STAR Collaboration), Phys. Lett. B 634, 347 (2006).

[13] K. Pearson, Phil. Trans. Royal Soc. 187, 253 (1896).

[14] T. A. Trainor and D. T. Kettler, Phys. Rev. D 74, 034012 (2006).

[15] D. Acosta D et al. (CDF Collaboration), Phys. Rev. D 68, 012003 (2003).

[16] W. Braunschweig et al. (TASSO Collaboration), Z. Phys. C 47, 187 (1990).

[17] M. Z. Akrawy et al. (OPAL Collaboration), Phys. Lett. B 247, 617 (1990).

[18] Yu. L. Dokshitzer, V. A. Khoze, A. H. Mueller and S. I. Troyan, Basics of perturbative QCD, Editions Frontières, Gif-sur-Yvette, (1991).

[19] J. Adams et al. (STAR Collaboration), Phys. Rev. Lett. 95, 152301 (2005)

[20] C. Adler et al. (STAR Collaboration), Phys. Rev. Lett. 90, 082302 (2003).

[21] R. W. Park, PhD Thesis, Dept. of Chemical Engineering, U. of Wisconsin, 1970.

[22] Ya. I. Azimov, Yu. L. Dokshitzer, V. A. Khoze, S. I. Troyan, Z. Phys. C 27, 65 (1985), Z. Phys. C 31, 213 (1986).

[23] J. Adams et al. (STAR Collaboration), Phys. Rev. C 73, 064907 (2006). 